\title{
Subfoveal serous retinal detachment associated with extramacular branch retinal vein occlusion
}

This article was published in the following Dove Press journal:

Clinical Ophthalmology

29 January 2013

Number of times this article has been viewed

\section{Toru Ota}

Akitaka Tsujikawa

Tomoaki Murakami

Ken Ogino

Yuki Muraoka

Kyoko Kumagai

Yumiko Akagi-Kurashige

Kazuaki Miyamoto

Nagahisa Yoshimura

Department of Ophthalmology and Visual Sciences, Kyoto University Graduate School of Medicine, Kyoto, Japan
Correspondence: Akitaka Tsujikawa Department of Ophthalmology and Visual Sciences, Kyoto University Graduate School of Medicine, Sakyo-ku, Kyoto 606-8507, Japan

Tel +81757513260

Fax +8I 757520933

Email tujikawa@kuhp.kyoto-u.ac.jp
Purpose: To study the pathophysiology of subfoveal serous retinal detachment (SRD) observed in eyes with extramacular branch retinal vein occlusion (BRVO).

Methods: We retrospectively reviewed the medical records of nine patients (nine eyes) with extramacular BRVO with macular complications that were examined using optical coherence tomography (OCT).

Results: Extramacular BRVO was observed in the inferior area in three eyes, in the superior area in five eyes, and in the nasal area in one eye. Visual acuity was moderately disturbed (median, 0.6; range, 0.2-0.9, measured using the Landolt chart). One eye showed extensive SRD that was connected to the area affected by BRVO through the subretinal space. In eight of the eyes, focal SRD was observed beneath the fovea without subretinal connections to the area affected by BRVO. However, all these eyes showed marked retinal swelling in the outer retina, particularly in the outer plexiform layer. In two of the eyes, detailed OCT examinations showed a small break on the external surface of the retina connecting the swollen outer retina with the underlying SRD. All eyes showed hyperreflective foci in the outer retina, most frequently along the inner boundary of the outer plexiform layer and external limiting membrane.

Conclusion: Extramacular BRVO is often accompanied by focal SRD beneath the fovea. Leakage from the retinal capillaries affected by BRVO travelled via the outer plexiform layer and caused SRD under the fovea.

Keywords: branch retinal vein occlusion, extramacular, macular edema, optical coherence tomography, retinal detachment

\section{Introduction}

Branch retinal vein occlusion (BRVO) is the second most common retinal vascular disorder after diabetic retinopathy. ${ }^{1,2}$ Acute BRVO is characterized by flame-shaped retinal hemorrhage, cotton-wool spots, and venous dilation in the affected retina. ${ }^{2,3}$ Increased intravascular pressure with reduced blood flow in the macular capillaries and the production of vascular endothelial growth factor from the ischemic retina lead to dysfunction of the endothelial blood-retinal barrier and the result is increased leakage in the macular area. ${ }^{4}$ When the fovea is involved in BRVO, visual acuity is often decreased due to macular edema. ${ }^{5,6}$ In addition, it is reported that macular edema associated with BRVO is often accompanied by serous retinal detachment (SRD) beneath the fovea and extensive leakage from macular capillaries may migrate to the subretinal space..$^{7-11}$

Patients with BRVO are often asymptomatic when the macular capillaries are not affected. However, extramacular BRVO occasionally causes visual disturbances due 
to macular complications, such as macular edema or foveal SRD. ${ }^{12-14}$ Previously, Wise and Wangvivat ${ }^{15}$ reported that the macula experiences an exaggerated SRD in some retinal disorders and Finkelstein and Patz ${ }^{16}$ reported the distant effects of peripheral BRVO on the macula. However, the reason why SRD in eyes with extramacular BRVO occurs preferentially beneath the fovea remains unknown. In the present study, we examined sequential sections obtained from spectral-domain optical coherence tomography (OCT) from eyes with extramacular BRVO and elucidated the pathophysiology of macular complications associated with extramacular BRVO.

\section{Patients and methods}

We retrospectively reviewed the medical records of nine patients (nine eyes; six men and three women, ranging in age from 53 to 88 years [average, $65.7 \pm 12.0$ years] [Table 1]) with extramacular BRVO with macular complications, who had been examined at the Department of Ophthalmology, Kyoto University Hospital, from October 2007 to November 2011. The current study was approved by the Institutional Review Board at Kyoto University Graduate School of Medicine and adhered to the tenets of the Declaration of Helsinki.

On their initial visits, patients underwent comprehensive ophthalmologic examinations, including measurement of bestcorrected visual acuity using the Landolt chart, determination of intraocular pressure, indirect ophthalmoscopy, slit-lamp biomicroscopy with a contact lens, and spectral-domain OCT (Spectralis HRA + OCT, Heidelberg Engineering, Heidelberg, Germany; 3D-OCT 1000, Topcon, Tokyo, Japan). The macular region was examined in detail with consecutive thin sections of OCT in order to evaluate morphologic changes in the macular area. Vertical and horizontal line scans, which were centered on the fovea, were also obtained. Using these OCT images, we performed three measurements: total retinal thickness in the fovea, thickness of the neurosensory retina in the fovea, and thickness of the SRD in the fovea.

\section{Results}

In the current study, the medical records of nine eyes from nine patients with extramacular BRVO were reviewed. The duration of symptoms ranged from 2 days to 1 year (median, 1 month). Extramacular BRVO was observed in the inferior area in three eyes, in the superior area in five eyes, and in the nasal area in one eye. The fovea was not directly affected by retinal hemorrhage, macular edema, or ischemia as a result of the BRVO. However, visual acuity was moderately disturbed

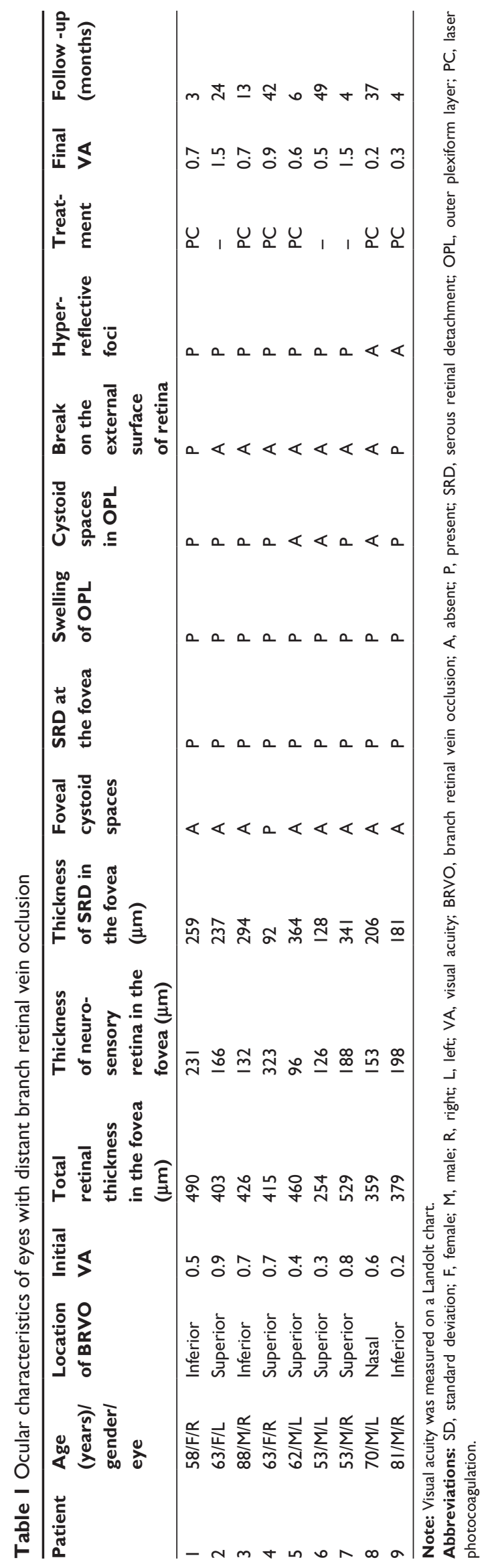


(median, 0.6; range, 0.2-0.9 according to the Landolt chart). Total retinal thickness in the fovea ranged from $254 \mu \mathrm{m}$ to $529 \mu \mathrm{m}$ (mean \pm standard deviation; $413 \pm 80 \mu \mathrm{m}$ ). In all eyes, OCT examinations showed SRD under the fovea, which was thickest beneath the fovea, ranging from $92 \mu \mathrm{m}$ to $364 \mu \mathrm{m}$ (mean \pm standard deviation; $234 \pm 92 \mu \mathrm{m}$ ). Most eyes retained the preserved convex of the fovea with a mean foveal thickness in the neurosensory retina of $179 \pm 68 \mu \mathrm{m}$.

Of these nine eyes, one showed extensive subfoveal SRD that was connected to the area affected by BRVO through the subretinal space. In the remaining eight eyes, OCT showed focal foveal SRD without subretinal connections to the area affected by BRVO. However, all of the eyes showed marked retinal swelling in the outer retina, particularly in the outer plexiform layer (Figure 1). Six eyes showed numerous cystoid spaces in the outer plexiform layer. In two of the eyes, detailed OCT examinations showed a small break on the external surface of the neurosensory retina that connects the swollen outer retina with the underlying SRD (Figure 2). All eyes showed hyperreflective foci in the outer retina, most frequently along the inner boundary of the outer plexiform layer and external limiting membrane (Figure 3 ). In the patients in the present study, none of the eyes showed vitreomacular traction in the macular area.

SRD regressed spontaneously in three of the eyes, and six of the eyes showed SRD regression immediately after the application of focal laser photocoagulation for BRVO (Figure 3). The mean follow-up duration was $20.2 \pm 18.3$ months. At the final visit, visual acuity ranged from 0.2 to 1.5 (median, 0.65 ), as measured by the Landolt chart.

\section{Discussion}

In the current study, nine eyes with extramacular BRVO showed retinal swelling in the macular area with foveal SRD. Macular swelling was predominantly observed in the outer plexiform layer while the inner layer of the retina often retained relatively normal physiologic structure. Using timedomain OCT, Takahashi et a ${ }^{14}$ previously reported four cases of foveal SRD associated with extramacular BRVO. The authors speculated that the leakage from the extramacular BRVO traveled to the macula through the subretinal space and formed the SRD beneath the fovea. In the current study, however, we could not detect any subretinal connections between the area affected by BRVO and the foveal SRD, even with sequential thin sectioning using spectral-domain OCT.

The eyes from two of the patients in the study showed a small break on the external surface of the detached neurosensory retina near the fovea. In these eyes, the break

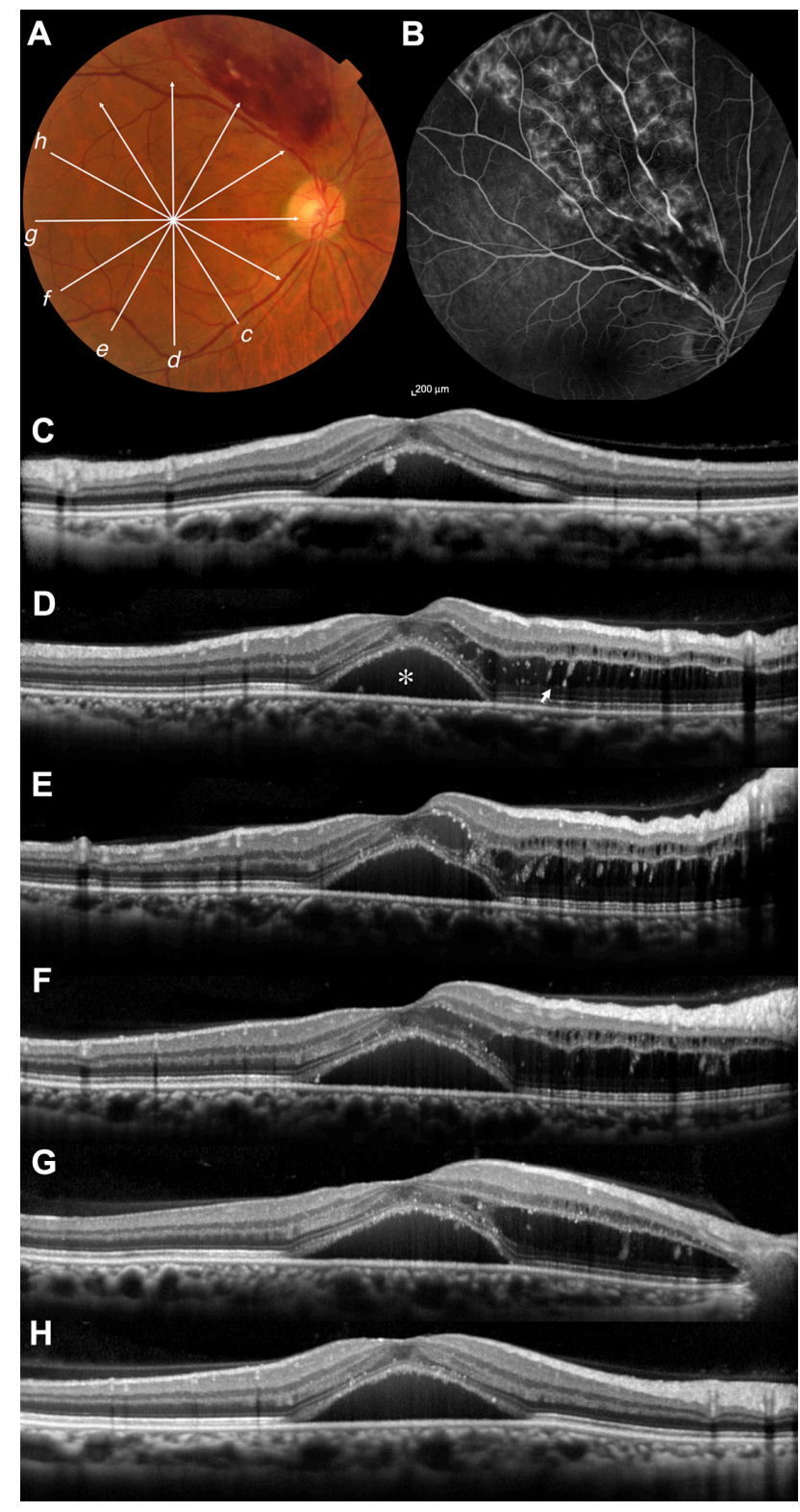

Figure I Extramacular branch retinal vein occlusion (BRVO) with serous retinal detachment beneath the fovea. (A) Fundus photograph shows a flame-shaped hemorrhage out of the vascular arcade (patient 7). Visual acuity was measured as 0.8 using the Landolt chart. (B) Fluorescein angiogram shows no leakage in the macular area. $(\mathbf{C}-\mathbf{H})$ Sectional images along the arrows $\mathrm{c}-\mathrm{h}$ in the fundus photograph were obtained using optical coherence tomography. Focal serous retinal detachment is seen under the fovea (asterisk). No subretinal connection is seen between the area affected with extramacular BRVO and the serous detachment. Marked retinal swelling in the outer retina, especially in the outer plexiform layer, is seen between the fovea and the area affected with BRVO. Numerous cystoid spaces are seen in the outer plexiform layer (arrow).

seemed to allow fluid that had accumulated within the outer retina to flow into the subretinal space. Recent advances in OCT resolution and contrast have facilitated the detection of breaks on the external surface of the neurosensory retina in eyes with diabetic macular edema, ${ }^{17}$ retinal arterial macroaneurysms, ${ }^{18}$ and retinal vein occlusions. ${ }^{10}$ Although it may be an uncommon feature associated with SRD, we 


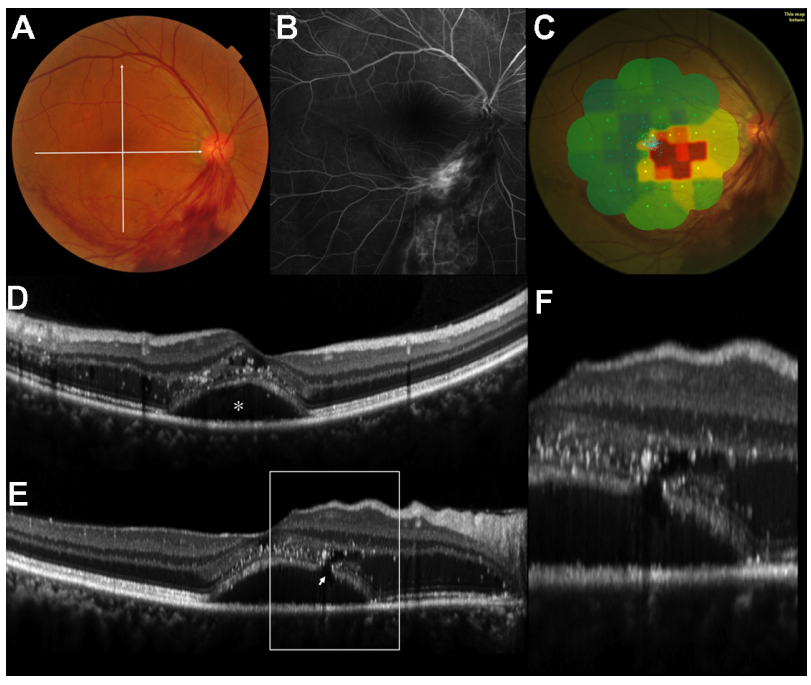

Figure $2 \mathrm{~A}$ small break on the external boundary of the retina in an eye with serous retinal detachment associated with extramacular branch retinal vein occlusion. (A) Fundus photograph shows a flame-shaped hemorrhage out of the vascular arcade (patient I). Visual acuity was measured as 0.5 using the Landolt chart. (B) Fluorescein angiogram shows no leakage in the macular area. (C) Microperimetry shows decreased retinal function in the macular area involving the fovea. Vertical (D) and horizontal (E) sectional images were obtained with optical coherence tomography along the white arrows. Extensive retinal edema and numerous hyperreflective foci are seen predominantly in the outer plexiform layer with a focal serous detachment beneath the fovea. A small break is seen on the external boundary of the detached retina. (F) Magnified image depicted in Figure 2E more clearly shows the aforementioned break (arrow). The swollen outer retina appears to be connected with the subretinal space.

could not detect any apparent breaks on the external surface of the retina in the remaining eyes. Small breaks on the external surface of the retina may be one possible mechanism for causing focal SRD beneath the fovea. Simple diffusion of intraretinal fluid into the subretinal space may represent a more common cause of foveal SRD associated with extramacular BRVO. ${ }^{19}$

It is not known why focal SRD associated with extramacular BRVO invariably occurs beneath the fovea. Previously, Taki et $\mathrm{al}^{20}$ reported a case of macular edema caused by an extramacular BRVO that improved after vitrectomy. However, none of the eyes from the patients reviewed in this study showed vitreomacular traction in the macular area. Tsujikawa et $\mathrm{al}^{10}$ reported that Müller cell cone traction plays a role in the formation of small pointed foveal SRD in eyes with retinal vein occlusion. They reported that small pointed SRD extends to become dome-shaped SRD, which resembles the SRD observed in our study patients. Müller cell cones, which are believed to serve as plugs that bind the photoreceptor cells to the foveola, ${ }^{21}$ extend outward and form the internal limiting membrane at the foveal area. ${ }^{22,23}$ In eyes with extramacular BRVO, leakage from the affected retinal capillaries travels preferentially through the outer plexiform layer and causes

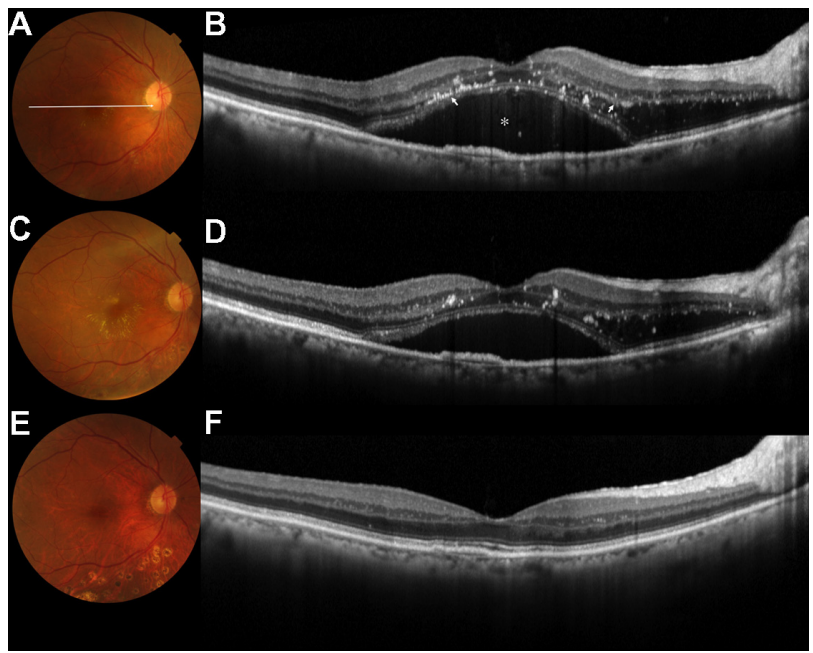

Figure 3 Regression of serous retinal detachment by focal laser photocoagulation to extramacular branch retinal vein occlusion (BRVO). (A) Fundus photograph shows residual retinal hemorrhage and shut vessels inferiorly out of the vascular arcade (patient 3). Hard exudate deposition is seen around the fovea. (B) Horizontal sectional image was obtained using optical coherence tomography along the white arrow. Extensive retinal edema is seen in the outer plexiform layer with a focal serous detachment beneath the fovea. Hyperreflective foci are predominantly seen along the inner boundary of the outer plexiform layer and external limiting membrane. Visual acuity was measured as 0.7 using the Landolt chart. Focal laser photocoagulation was applied to the area affected with extramacular BRVO. (C) Three weeks after treatment, the hard exudate around the fovea seemed to increase. (D) Horizontal sectional image along the white arrow shows a decrease in serous retinal detachment. (E) One year after the treatment, the hard exudate disappeared completely. (F) Horizontal sectional image along the white arrow shows a complete absorbance of macular edema and serous retinal detachment. Final visual acuity was measured as 0.9 using the Landolt chart.

retinal edema mainly within Henle's layer. At the fovea, however, Müller cell cones serve to maintain the integrity of the foveal structure and consequently cause traction with the inner and outer segments of the foveal photoreceptors, which results in foveal SRD. In the current study, none of the records reviewed reported focal SRD outside the fovea. Because patients may not have had symptoms arising from the focal SRD formed outside the fovea, we cannot deny the possibility that some patients had this type of SRD.

Of the nine eyes in this study, two had a final visual acuity of less than 0.5. Visual prognosis of eyes with extramacular BRVO is better than those with macular BRVO, even if they have SRD under the fovea. In the current study, the mean total retinal thickness in the fovea was $413 \pm 80 \mu \mathrm{m}$. However, most of the patients reviewed had relatively normal neurosensory retinal thickness in the fovea (mean \pm standard deviation; $179 \pm 68 \mu \mathrm{m})$. The formation of SRD under the fovea may contribute to retaining the integrity of the foveal structure. Unless SRD develops under the fovea, fluid extending into the fovea may form large foveal cystoid spaces, leading to severe visual dysfunction. ${ }^{24}$ SRD may occasionally regress spontaneously, but focal laser photocoagulation often results 
in immediate regression. In the current study, all eyes showed hyperreflective foci in the outer retina, most frequently along the inner boundary of the outer plexiform layer and external limiting membrane. It is reported that hyperreflective foci are mainly small lipid exudates left after the absorption of intraretinal fluid. ${ }^{25,26}$ Foveal accumulation of the hard exudate may cause destruction of the foveal structure and lead to poor visual prognosis. ${ }^{13}$

Limitations of the current study include its retrospective nature and small sample size. In addition, when extramacular BRVO causes no macular complications, patients rarely present ocular symptoms. Thus, it is difficult to estimate the risk of macular complications when BRVO occurs outside the vascular arcade. Furthermore, in all of our patients, leakage from the BRVO extended towards the macula, even if BRVO occurred in the inferior quadrant. The reason for this preferential fluid extension is unclear. Therefore, although the leakage from extramacular BRVO may extend in various directions, we only reviewed cases with extramacular BRVO that had macular complications.

\section{Disclosure}

The authors have no financial interest in the materials or devices mentioned in this article.

\section{References}

1. Wong TY, Scott IU. Clinical practice. Retinal-vein occlusion. $N$ Engl J Med. 2010;363(22):2135-2144.

2. Jonas J, Paques M, Mones J, Glacet-Bernard A. Retinal vein occlusions. Dev Ophthalmol. 2010;47:111-135.

3. Cruz-Villegas V, Puliafito CA, Fujimoto FG. Retinal Vascular Diseases, 2nd ed. In: Schuman JS, Puliafito CA, Fujimoto FG, editors. Optical Coherence Tomography of Ocular Disease. Thorofare: SLACK, 2004:103-156.

4. Battaglia Parodi M, Isola V. Branch retinal vein occlusion and exudative retinal detachment: pathogenetical aspects. Ophthalmologica. 1994;208(1):29-31.

5. Glacet-Bernard A, Coscas G, Chabanel A, Zourdani A, Lelong F, Samama MM. Prognostic factors for retinal vein occlusion: prospective study of 175 cases. Ophthalmology. 1996;103(4):551-560.

6. The Branch Vein Occlusion Study Group. Argon laser photocoagulation for macular edema in branch vein occlusion. Am J Ophthalmol. 1984; 98(3):271-282.

7. Schatz H, Yannuzzi L, Stransky TJ. Retinal detachment secondary to branch vein occlusion: Part II. Ann Ophthalmol. 1976;8(12): 1461-1471.

Clinical Ophthalmology

\section{Publish your work in this journal}

Clinical Ophthalmology is an international, peer-reviewed journal covering all subspecialties within ophthalmology. Key topics include: Optometry; Visual science; Pharmacology and drug therapy in eye diseases; Basic Sciences; Primary and Secondary eye care; Patient Safety and Quality of Care Improvements. This journal is indexed on Submit your manuscript here: http://www.dovepress.com/clinical-ophthalmology-journal
8. Schatz H, Yannuzzi L, Stransky TJ. Retinal detachment secondary to branch vein occlusion: Part I. Ann Ophthalmol. 1976;8(12): $1437-1452$.

9. Spaide RF, Lee JK, Klancnik JK Jr, Gross NE. Optical coherence tomography of branch retinal vein occlusion. Retina. 2003;23(3): 343-347.

10. Tsujikawa A, Sakamoto A, Ota M, et al. Serous retinal detachment associated with retinal vein occlusion. Am J Ophthalmol. 2010;149(2): 291-301. e295.

11. Yamaguchi Y, Otani T, Kishi S. Serous macular detachment in branch retinal vein occlusion. Retina. 2006;26(9):1029-1033.

12. Otani T, Yamaguchi Y, Kishi S. Serous macular detachment secondary to distant retinal vascular disorders. Retina. 2004;24(5):758-762.

13. Takahashi K, Kashima T, Kishi S. Massive macular hard exudates associated with branch retinal vein occlusion. Jpn J Ophthalmol. 2005;49(6):527-529.

14. Takahashi K, Kishi S. Serous macular detachment associated with midperipheral branch retinal vein occlusion. Retina. 2004;24(2): 299-301.

15. Wise GN, Wangvivat $Y$. The exaggerated macular response to retinal disease. Am J Ophthalmol. 1966;61(5 Pt 2):1359-1363.

16. Finkelstein D, Patz A. Distant effect of peripheral branch vein occlusion on the macula. Trans Am Ophthalmol Soc. 1988;86:380-388.

17. Ota M, Nishijima K, Sakamoto A, et al. Optical coherence tomographic evaluation of foveal hard exudates in patients with diabetic maculopathy accompanying macular detachment. Ophthalmology. 2010;117(10): 1996-2002.

18. Tsujikawa A, Sakamoto A, Ota M, et al. Retinal structural changes associated with retinal arterial macroaneurysm examined with optical coherence tomography. Retina. 2009;29(6):782-792.

19. Marmor MF. Mechanisms of fluid accumulation in retinal edema. Doc Ophthalmol. 1999;97(3-4):239-249.

20. Taki W, Oohira A, Hirakata A. Macular edema from distant branch retinal vein occlusion improved after vitrectomy. Jpn J Ophthalmol. 2009;53(2):184-186.

21. Hangai M, Ojima Y, Gotoh N, et al. Three-dimensional Imaging of Macular Holes with High-speed Optical Coherence Tomography. Ophthalmology. 2007;114(4):763-773.

22. Gass JDM. Müller cell cone, an overlooked part of the anatomy of the fovea centralis: hypotheses concerning its role in the pathogenesis of macular hole and foveomacular retinoschisis. Arch Ophthalmol. 1999;117(6):821-823.

23. Yamada E. Some structural features of the fovea centralis in the human retina. Arch Ophthalmol. 1969;82(2):151-159.

24. Murakami T, Tsujikawa A, Ohta M, et al. Photoreceptor status after resolved macular edema in branch retinal vein occlusion treated with tissue plasminogen activator. Am J Ophthalmol. 2007; 143(1):171-173.

25. Ogino K, Murakami T, Tsujikawa A, et al. Characteristics of optical coherence tomographic hyperreflective foci in retinal vein occlusion. Retina. 2012;32(1):77-85.

26. Bolz M, Schmidt-Erfurth U, Deak G, Mylonas G, Kriechbaum K, Scholda C. Optical coherence tomographic hyperreflective foci a morphologic sign of lipid extravasation in diabetic macular edema Ophthalmology. 2009;116(5):914-920.

PubMed Central and CAS, and is the official journal of The Society of Clinical Ophthalmology (SCO). The manuscript management system is completely online and includes a very quick and fair peer-review system, which is all easy to use. Visit http://www.dovepress.com/ testimonials.php to read real quotes from published authors. 